\title{
1 Real-time luminescence assay for cytoplasmic cargo delivery of extracellular
}

\section{2 vesicles}

3

4 Masaharu Somiya ${ }^{*}$ and Shun'ichi Kuroda

5 The Institute of Scientific and Industrial Research, Osaka University, Osaka 567-0047,

6 Japan

7

8 *Corresponding author: Prof. Masaharu Somiya, Ph.D.

9 Department of Biomolecular Science and Reaction, The Institute of Scientific and

10 Industrial Research, Osaka University, 8-1 Mihogaoka, Ibaraki, Osaka 567-0047, Japan.

11 E-mail: msomiya@sanken.osaka-u.ac.jp

12 Phone: 81-6-6879-8462

13

14 


\section{Abstract}

16 Extracellular vesicles (EVs) have been considered to deliver biological cargos between

17 cells and mediate intercellular communication. However, the mechanisms that underlie

18 the biological process of EV uptake and cytoplasmic cargo release in recipient cells are

19 largely unknown. Quantitative and real-time assays for assessment of the cargo delivery

20 efficiency inside recipient cells have not been feasible. In this study, we developed an

21 EV cargo delivery (EVCD) assay using a split luciferase called the NanoBiT system.

22 Recipient cells expressing LgBiT, a large subunit of luciferase, emit luminescence when

23 the EV cargo proteins fused with a small luminescence tag (HiBiT tag) that can

24 complement LgBiT are delivered to the cytoplasm of recipient cells. Using the EVCD

25 assay, the cargo delivery efficiency of EVs could be quantitatively measured in real

26 time. This assay was highly sensitive in detecting a single event of cargo delivery per

27 cell. We found that modification of EVs with a virus-derived fusogenic protein

28 significantly enhanced the cytoplasmic cargo delivery; however, in the absence of a

29 fusogenic protein, the cargo delivery efficiency of EVs was below the threshold of the

30 assay. The EVCD assay could assess the effect of entry inhibitors on EV cargo delivery.

31 Furthermore, using a luminescence microscope, the cytoplasmic cargo delivery of EVs 
32 was directly visualized in living cells. This assay could reveal the biological mechanism

33 of the cargo delivery processes of EVs.

34

35 Keywords: cargo transfer; extracellular vesicles; membrane fusion; NanoBiT; VSV-G 


\section{$37 \quad$ Introduction}

38 Extracellular vesicles (EVs), membranous nanoparticles secreted by living cells,

39 are thought to be involved in intercellular communication in various species from

40 microorganisms to vertebrate ${ }^{1,2}$. Since EVs contain cargo molecules such as RNAs and

41 proteins in their luminal space, they may deliver the cargo molecules into recipient cells

42 and regulate biological functions in the recipient cells. Numerous studies have shown

43 that the treatment of recipient cells with EVs containing specific cargos (especially

44 microRNAs or proteins) results in phenotypic changes in the recipient cells. Owing to

45 the delivery capability of biomolecules, EVs have been studied as a promising drug

46 delivery system for therapeutic proteins or RNAs ${ }^{3,4}$.

47 However, the cargo delivery mechanism of EVs, especially the process of

48 cytoplasmic cargo release, remains largely unknown ${ }^{5}$. Mechanistically, EVs are mainly

49 endocytosed by recipient cells, fuse with the endosomal/lysosomal membrane, and

50 release their cargo into the cytoplasm ${ }^{5,6}$. Although few studies have shown that EVs are

51 capable of fusing with the cellular membrane of recipient cells ${ }^{7,8}$, direct evidence

52 indicating the cytoplasmic cargo delivery of EVs has not been demonstrated.

53 We discussed the possibility of "EV cargo transfer hypothesis" in our previous

54 review and concluded that cargo delivery by EVs might not be a frequent event as 
55 generally accepted ${ }^{9}$. Several studies have suggested that EV-mediated cargo delivery is

56 a rare event. When the recipient cells are treated with EVs in vitro, only $0.1 \%$ to $5.0 \%$

57 of the cell population exhibit the functional readout of cargo delivery, although the

58 efficacy depends on the experimental system ${ }^{10-12}$.

59 To decipher the mechanism and physiological relevance of EV cargo delivery,

60 a feasible and reliable assay to measure cargo delivery in real time is necessary.

61 Conventionally, cargo delivery of EVs is evaluated by the phenotypic change in the

62 recipient cells, although these methods are often interfered with the experimental

63 artifacts that can be induced by contaminants in the EV fraction ${ }^{13}$. Another approach

64 for the assessment of EV cargo delivery involves use of reporter assays for measuring

65 functional miRNA activity in recipient cells ${ }^{14}$. This assay is based on the assumption

66 that EV-mediated delivery of miRNA leads to a change in reporter gene expression in

67 recipient cells. However, this assay could not demonstrate direct evidence of cargo

68 transfer by EVs because of several confounding factors ${ }^{9}$.

69 In this study, we developed a quantitative and real-time luminescence assay to

70 measure cargo protein delivery by EVs in recipient cells. The key feature of this EV

71 cargo delivery (EVCD) assay is the luciferase complementation assay using Oplophorus

72 gracilirostris-derived highly bright luciferase (NanoLuc) ${ }^{15,16}$. A small fragment of 
73 NanoLuc (HiBiT tag) was fused to EV cargo proteins, while the large subunit of

74 NanoLuc (LgBiT) was expressed in recipient cells. When the HiBiT-tagged cargo

75 proteins are delivered to the cytoplasm of recipient cells, luciferase fragments

76 complement and emit luminescence signals (Fig. 1A). Since the complemented

77 NanoLuc is much brighter than conventional luciferases such as firefly or Renilla

78 luciferases, NanoLuc-based assays are sensitive enough to detect the rare event of EV

79 cargo delivery. Furthermore, this assay enabled us to measure the kinetics of cargo

80 protein delivery by EVs and to visualize the cytoplasmic cargo delivery by EVs in real

81 time.

82 


\section{Results and Discussion}

\section{Characterization of HiBiT-tagged EV cargo proteins}

85 The EVCD assay (Fig. 1A) is based on the complementation of HiBiT and LgBiT in the

86 cytoplasm. When the EVs containing HiBiT-tagged cargo are delivered to the

87 cytoplasm of LgBiT-expressing recipient cells, emitted luminescence can be detected.

88 To establish the EVCD assay, we first attempted to tag the EV cargos with HiBiT (Fig.

89 1B). Three types of protein EV cargos including EGFP, a self-assembling protein

90 EPN-01 ${ }^{17}$, and tetraspanins were used. The first cargo EGFP was tagged at the

91 N-terminal with HiBiT and overexpressed in the donor cells. Cytoplasmic EGFPs may

92 be passively loaded into EVs. The second cargo EPN-01 was a nanocage-forming

93 protein that was designed de novo and secreted from cells via the ESCRT pathway with

94 EVs ${ }^{17}$. The original Myc-tag of this cargo was replaced with an HiBiT tag.

95 Tetraspanins, typical EV marker proteins embedded in the EV membrane, were also

96 tagged with HiBiT at their N-termini.

97 All HiBiT-tagged proteins were expressed in HEK293T cells, and expression

98 levels were measured by mixing cell lysates with LgBiT and NanoLuc substrates (Fig.

99 1C). EGFP and EPN-01showed higher expression levels compared to tetraspanins.,

100 Expression of HiBiT-tagged EGFP and EPN-01 was confirmed by western blotting 
101 using LgBiT as a probe protein (Fig. 1D). These results confirmed that EGFP and

102 EPN-01 had feasibility in the EVCD assay.

103 We assessed whether HiBiT-tagged cargo proteins were encapsulated in EVs by

104 immunoprecipitation (Fig. S1). EVs in the culture supernatant were immunoprecipitated

105 using antibodies targeting CD81, a typical EV marker (Fig. S1A), and vesicular

106 stomatitis virus glycoprotein (VSV-G), a fusogenic viral membrane protein that was

107 incorporated into EVs (Fig. S1B). When the supernatant of the cargo protein-expressing

108 cells was immunoprecipitated using anti-CD81 antibodies, cargo proteins were

109 precipitated, indicating that cargo proteins were encapsulated inside EVs (Fig. S1C and

110 S1D). Furthermore, anti-VSV-G antibody could enrich HiBiT-tagged proteins

111 co-expressed with VSV-G, suggesting the incorporation of VSV-G in the EV membrane

$112{ }^{17}$ and encapsulation of HiBiT-tagged cargo proteins (Fig. S1C and S1E).

113 Immunoprecipitation was strongly abrogated by detergent treatment (Fig. S1F),

114 indicating that the HiBiT-tagged cargo protein was encapsulated in VSV-G ${ }^{+}$and/or

$115 \mathrm{CD}^{+} 1^{+}$membrane vesicles.

116 Generally, the amount of EVs in the supernatant is low; therefore, a

117 concentration process is necessary to acquire a sufficient amount of EVs for the assay.

118 In this study, EVs containing HiBiT-tagged proteins were concentrated by 
119 poly(ethylene glycol) (PEG) precipitation, which is a feasible concentration method for

120 small-scale purification ${ }^{18}$. As shown in Table 1, HiBiT-tagged EPN-01 was enriched

121 more than 10-fold by PEG precipitation, whereas HiBiT-tagged EGFP was not enriched.

122 This result indicated that a large fraction of EPN-01 in the supernatant was encapsulated

123 within EVs, while the majority of EGFPs were not encapsulated in EVs. Concentrated

124 EV fraction contained VSV-G and EV marker proteins (CD9, CD63, and CD81),

125 suggested that PEG precipitation successfully concentrated the VSV-G-displaying EVs

126 (Fig. 1E).

127

128

129 
130 Table 1 PEG precipitation of EVs encapsulating HiBiT-tagged protein cargo $(\mathrm{N}=3$,

131 mean \pm SD)

\begin{tabular}{|c|c|c|c|}
\hline \multicolumn{2}{|c|}{ Transfection } & \multirow{2}{*}{$\begin{array}{c}\text { HiBiT enrichment }(-)^{1} \\
12.4 \pm 3.4\end{array}$} & \multirow{2}{*}{$\frac{\text { HiBiT yield (\%) }}{27.6 \pm 7.6}$} \\
\hline & - & & \\
\hline & + VSV-G & $12.4 \pm 1.4$ & $27.5 \pm 3.1$ \\
\hline \multirow{2}{*}{ EGFP } & - & $0.3 \pm 0.1$ & $3.3 \pm 2.5$ \\
\hline & + VSV-G & $0.5 \pm 0.3$ & $6.0 \pm 5.4$ \\
\hline
\end{tabular}

134

135

136 
A

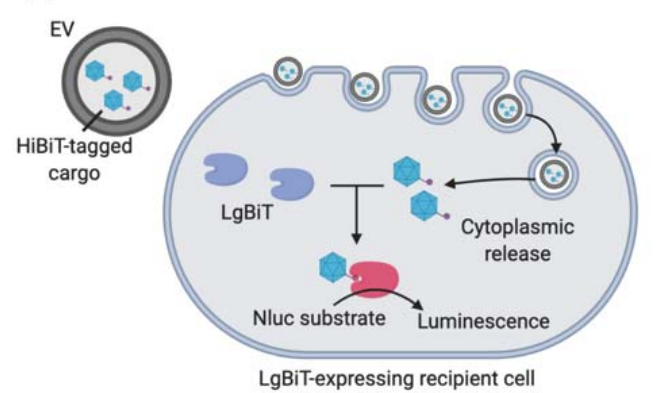

C

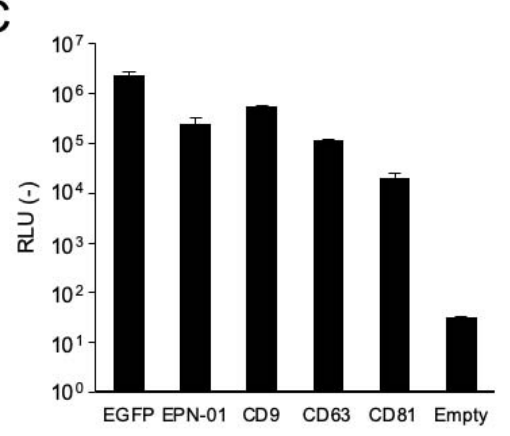

B

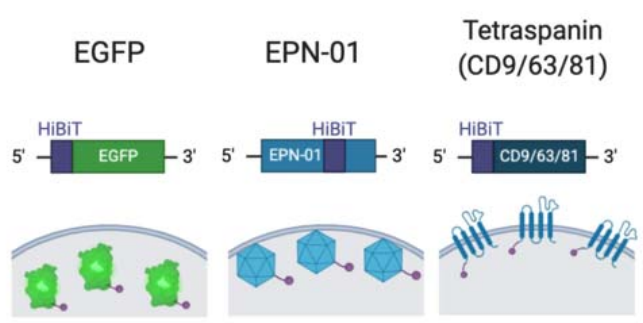

E PEG-EV

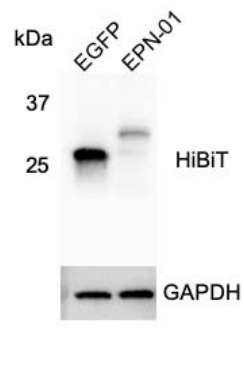

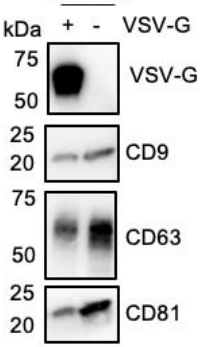

138 Fig. 1 Summary of the EVCD assay and characterization of HiBiT-tagged EV cargo proteins

140 (A) Schematic representation of the EVCD assay. EV containing HiBiT-tagged cargo is

141 internalized by LgBiT-expressing recipient cells, followed by cytoplasmic release of the

142 cargo. Spontaneous complementation of HiBiT-tagged protein cargo with LgBiT leads

143 to the elicitation of luminescence. (B) Schematic representation of HiBiT-tagged

144 proteins. Upper panels show the structure of expression plasmids. Lower panels show

145 the protein localization inside EVs. (C) Expression levels of HiBiT-tagged EV cargo

146 proteins in donor HEK293T cells. $\mathrm{N}=3$, mean \pm SD. (D) Detection of HiBiT-tagged 
147 EGFP and EPN-01 in cell lysate of transfected HEK293T cells using LgBiT as a probe.

148 GAPDH was used as a loading control. (E) Detection of VSV-G and EV marker

149 proteins in purified EV fraction. Culture supernatant was concentrated by PEG

150 precipitation and subjected to western blotting

152 Notably, substantial amounts of non-encapsulated HiBiT-tagged proteins (both

153 EPN-01 and EGFP) were present in the resultant EV fraction that could interfere with

154 the EVCD assay. Therefore, in the subsequent EVCD assay, it is mandatory to use a

155 DrkBiT peptide that complements and inactivates the luciferase activity of LgBiT to

156 competitively block the non-encapsulated HiBiT-tagged proteins (see below).

158 Real-time EVCD assay

159 We first estimated the sensitivity of the EVCD assay using a synthetic HiBiT peptide.

160 After lysis of approximately $1.0 \times 10^{5}$ LgBiT-expressing HEK293T cells, an HiBiT

161 peptide and a NanoLuc substrate were added to the lysate and luminescence signal was

162 measured (Fig. 2A). Approximately $0.1 \mathrm{fmol}$ of the HiBiT peptide was detected, 
163 suggesting that the assay was capable of measuring remarkably less amounts of

164 cytoplasmic cargo in the recipient cells.

165 Next, we measured the cargo delivery kinetics of EVs containing either

166 HiBiT-tagged EGFP or EPN-01. The first observation of EPN-01-containing EVs in the

167 EVCD assay demonstrated no luminescence signal within 90 min (Fig. 2B). For

168 controls, we used the EVs displaying VSV-G proteins, which confer EVs with

169 fusogenic activity that facilitates the cargo delivery of EVs by membrane fusion

170 between the EV and cellular membranes ${ }^{17,19}$. Evidently, VSV-G-displaying EVs

171 induced a gradual increase in the luminescence signal (Fig. 2C), suggesting that the

172 HiBiT-tagged EPN-01 was delivered to the cytoplasm and the presence of membrane

173 fusion proteins such as VSV-G was indispensable for achieving substantial cargo

174 delivery. The luminescence signal was observed as soon as 20 min after the addition of

175 EVs (Fig. 2D), suggesting that VSV-G could induce prompt fusion and release of

176 EPN-01 cargo into the cytoplasm. This result was consistent with that of previous

177 studies showing that the internalization and fusion of VSV was a rapid process, within 3

$178 \min$ in HeLa cells ${ }^{20}$ and 20 min in BHK cells ${ }^{21}$.

179 As described above, the concentrated EV fraction contains a substantial amount

180 of HiBiT-tagged proteins outside of EVs. Moreover, LgBiT may be leaked from the 
181 recipient cells into the medium ${ }^{22}$. These components significantly affect the sensitivity

182 and accuracy of the EVCD assay. As shown in Fig. S2, in the absence of DrkBiT, a

183 sudden increase in the luminescence signal was observed immediately after the addition

184 of EVs (Fig. S2A). However, in the presence of $1 \mu \mathrm{M}$ DrkBiT in the buffer,

185 luminescence signal emitted by EV-mediated cargo delivery was distinguishable from

186 non-specific luminescence signal (Fig. S2B), indicating that nonspecific

187 complementation of $\mathrm{LgBiT}$ and $\mathrm{HiBiT}$ outside the recipient cells interfered with the

188 assay. Therefore, it is mandatory to use a DrkBiT peptide in the EVCD assay to avoid

189 non-specific background signals.

190 To validate the EVCD assay and exclude an experimental artifact, we used

191 mutant VSV-G(P127D) that is incapable of fusing with the host cell membrane ${ }^{23}$.

192 Using both EGFP and EPN-01 as cargos, VSV-G(P127D) decreased the cargo delivery

193 efficacy of EVs compared to the parental VSV-G (Fig. 2D and 2E), which was

194 consistent with the findings of a previous report demonstrating that the fusogenic

195 activity of VSV-G was indispensable for cytoplasmic delivery of the EV cargo ${ }^{17,24}$.

196 These results support that the EVCD assay can elucidate the fusion and cytoplasmic

197 cargo release of EVs in recipient cells. 

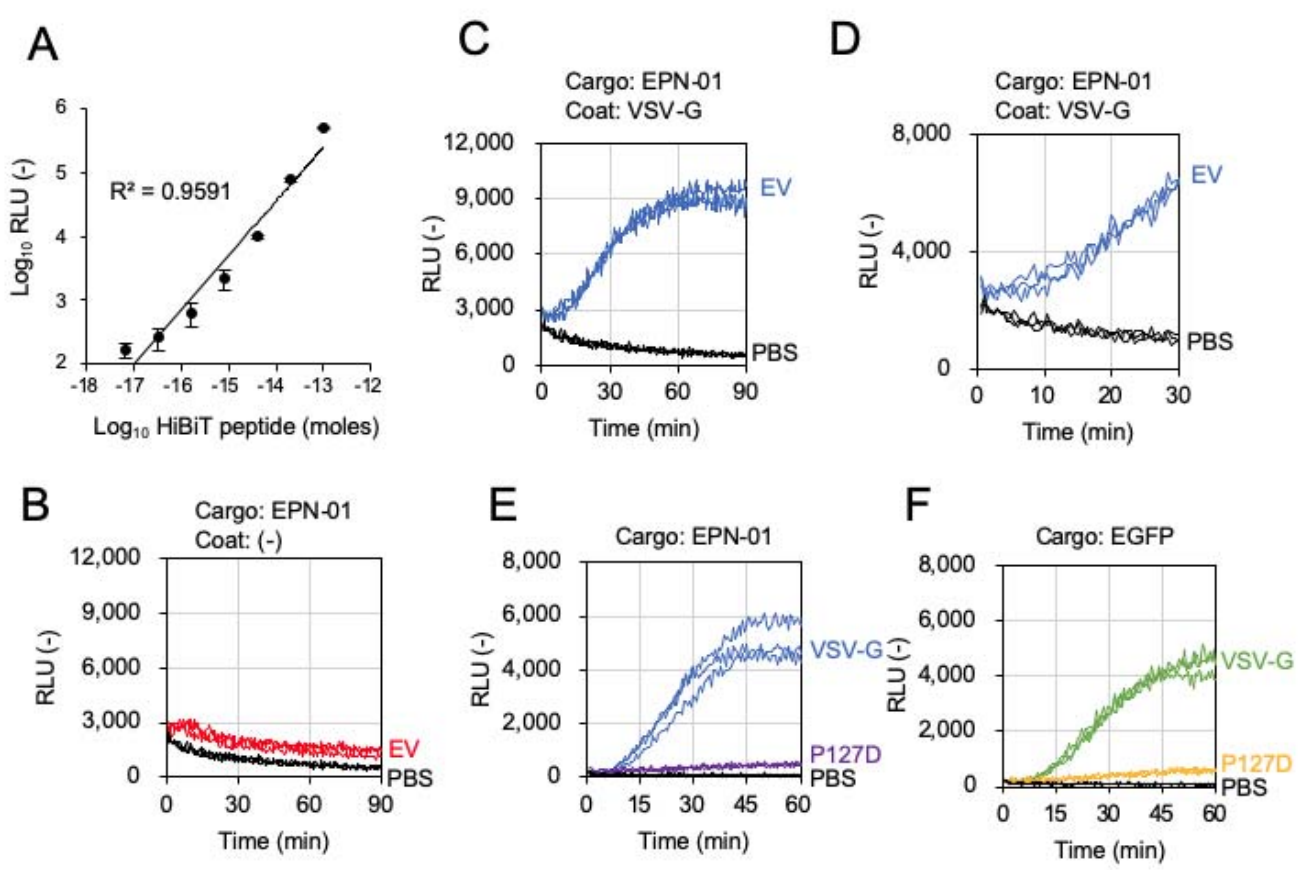

198

199 Fig. 2 EV cargo delivery (EVCD) assay

200 (A) Quantitative curve of the HiBiT peptide in cell lysate of LgBiT-expressing

201 HEK293T. N=3, mean \pm SD. (B) EVCD assay using EPN-01-containing EVs without

202 fusogenic protein. (C) EVCD assay using EPN-01-containing EVs with fusogenic

203 protein VSV-G. (D) Enlargement of (C) from 0 to 30 min. (E) EVCD assay using

204 EPN-01-containing EVs with either VSV-G or VSV-G(P127D). (F) EVCD assay using

205 EGFP-containing EVs with either VSV-G or VSV-G(P127D). PBS was used as a

206 negative control. All kinetics data represent information obtained from experiments

207 conducted in triplicate. 
209 Evaluation of EV entry inhibitors using the EVCD assay

210 We evaluated the effect of compounds that are known to inhibit endocytosis and

211 membrane fusion by using the EVCD assay with EPN-01-containing EVs modified with

212 VSV-G. Chlorpromazine ${ }^{25}$, Dynasore ${ }^{26}$, EIPA ${ }^{27}$, and Pitstop $2^{28}$ have been known to

213 inhibit the endocytosis of EVs, and all these compounds could significantly decrease the

214 cargo delivery of EVs (Fig. 3A-3D). Furthermore, chloroquine ${ }^{29}$ and bafilomycin A1 ${ }^{30}$,

215 both known to inhibit low pH-dependent fusion activity of VSV-G, abolished the cargo

216 delivery of EVs in a dose-dependent manner (Fig. 3E and 3F). These results confirmed

217 that the EVCD assay could evaluate the cargo delivery efficiency of EVs and the effect

218 of inhibitors. 

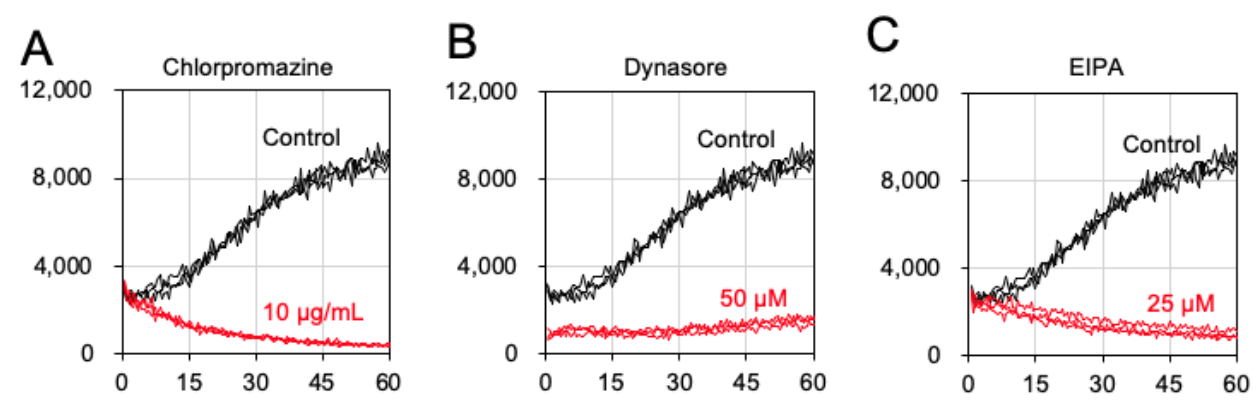

219
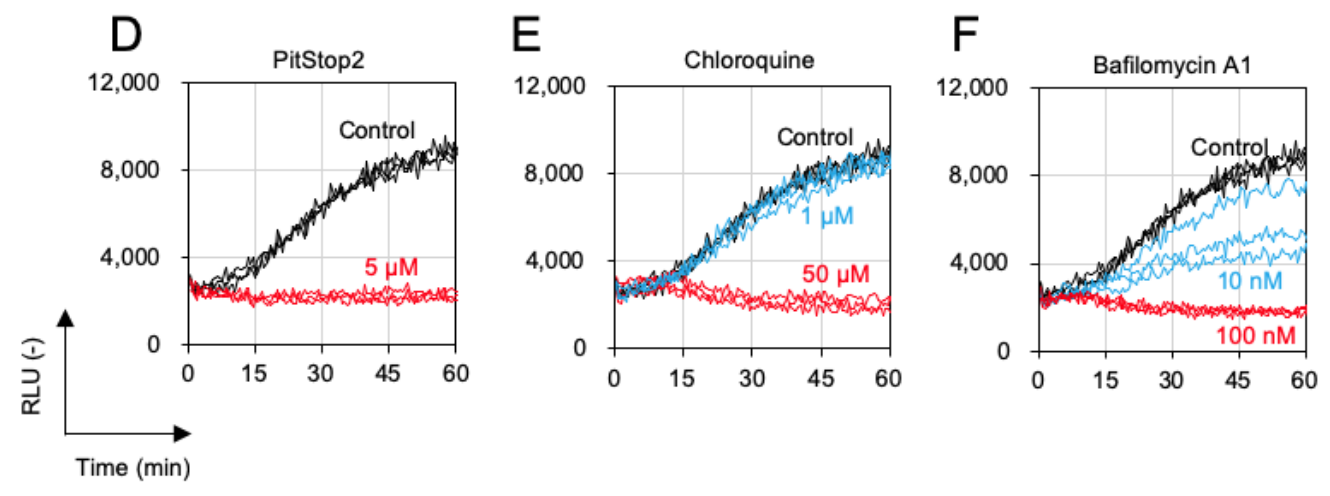

220 Fig. 3 The inhibitory effect of compounds on cargo delivery by EVs

221 Endocytosis inhibitors (chlorpromazine [A], Dynasore [B], EIPA [C], and Pitstop 2 [D])

222 and membrane fusion inhibitors (chloroquine $[\mathrm{E}]$ and bafilomycin $\mathrm{A} 1[\mathrm{~F}]$ ) were analyzed

223 in the EVCD assay using EPN-01-containing EVs modified with VSV-G. All kinetics

224 data represent information obtained from experiments conducted in triplicate.

226 The use of the EVCD assay to decipher the endosomal escape efficiency of EVs

227 It has been reported that endosome-destabilizing reagents such as chloroquine and

228 UNC10217832A can enhance the cargo delivery of EVs ${ }^{31}$. To confirm the effect of the 
229 endosomolytic reagent on the cargo delivery efficiency of EVs without VSV-G

230 modification, we evaluated whether chloroquine could enhance the cargo delivery of

231 EVs using the EVCD assay. Unexpectedly, chloroquine did not enhance the cargo

232 EPN-01 delivery by EVs (Fig. 4A) for 90 min. Conversely, cargo delivery of

233 VSV-G-modified EVs was significantly reduced by chloroquine (Fig. 3E and 4B),

234 suggesting that chloroquine increased the $\mathrm{pH}$ within endosomes/lysosomes in recipient

235 cells and inhibited membrane fusion by VSV-G.

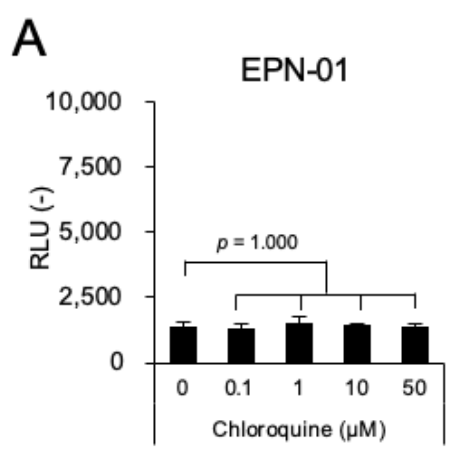

B

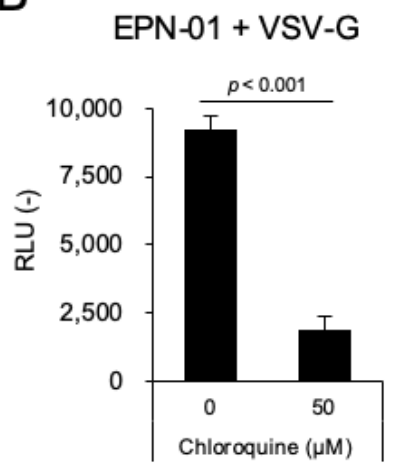

Fig. 4 Cargo delivery efficiency of

EPN-01-containing EVs in the presence of chloroquine

(A) EVs without VSV-G and (B) EVs with VSV-G.

Luminescence signal after 90 min of EV treatment

was represented. $\mathrm{N}=3$, mean $\pm \mathrm{SD}$. Statistical analysis was performed using one-way ANOVA followed by post hoc Dunnett's test (A) and the Student's $t$-test 
246 (B).

249 Real-time imaging of cytoplasmic cargo delivery by EVs

250 Imaging of the real-time cytoplasmic delivery of cargo molecules in recipient cells is of

251 prime importance, as the localization and timing of cargo delivery of EVs is largely

252 unknown. The EVCD assay described above can analyze a considerable segment of the

253 event of cargo delivery in a cell population with high sensitivity. Therefore, we

254 attempted to observe the luminescence signal emitted by cargo delivery at the

255 single-cell level using a luminescence microscope. We succeeded in capturing the

256 cytoplasmic cargo release of VSV-G-containing EVs in LgBiT-expressing HEK293T

257 cells (Fig. 5A to 5C and Supplementary Video). As shown in Fig. 5B and 5C,

258 luminescence dots were observed within recipient cells over time, suggesting that

259 EPN-01 nanocages were anchored to the cytoplasmic leaflet of the endo/lysosomal

260 membrane as an intact nanocage (Fig. 5D) because of the N-terminal myristoyl group of

261 EPN-01 that could anchor the membrane organelle ${ }^{17}$. 
bioRxiv preprint doi: https://doi.org/10.1101/2020.10.16.341974; this version posted January 13,2021 . The copyright holder for this preprint (which was not certified by peer review) is the author/funder. All rights reserved. No reuse allowed without permission.

263 


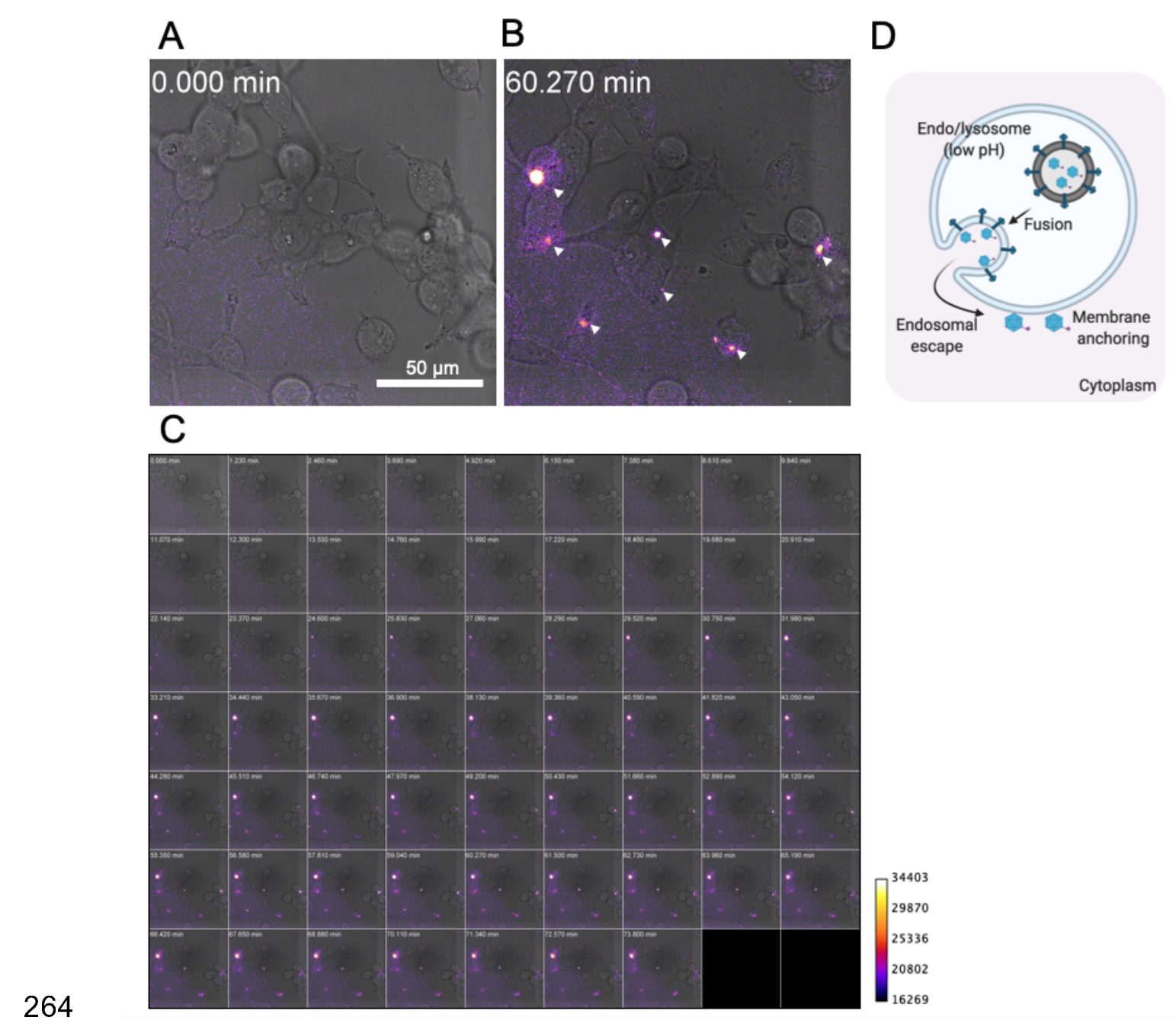

265 Fig. 5 Live cell imaging of cargo delivery of EVs with VSV-G

266 Luminescence images of recipient HEK293T cells before (A) and after $60 \mathrm{~min}(\mathrm{~B})$ of

267 treatment with EPN-01-containing EVs modified with VSV-G. Arrowheads indicate

268 complemented NanoLuc-derived luminescence signals within cells. (C) Series of

269 luminescence images of cells treated with EPN-01-containing EVs modified with

270 VSV-G from 0 to $73.8 \mathrm{~min}$ (see Supplementary Video). EVs were added at $0 \mathrm{~min}$. (D) 
bioRxiv preprint doi: https://doi.org/10.1101/2020.10.16.341974; this version posted January 13, 2021. The copyright holder for this preprint (which was not certified by peer review) is the author/funder. All rights reserved. No reuse allowed without permission.

271 Expected intracellular localization of EPN-01 after the fusion of EVs and cytoplasmic

272 release.

273 


\section{Conclusions}

275 In this study, we developed a novel assay to measure the real-time cargo delivery

276 efficiency of EVs in living recipient cells. Previously, the NanoBiT technology has been

277 used to evaluate viral entry ${ }^{22,32,33}$ and cytoplasmic drug delivery by polymeric

278 nanomaterials ${ }^{34}$. Toribio et al. demonstrated that split EGFP-luciferase fusion proteins

279 could be used to measure the cellular uptake of EVs ${ }^{35}$. However, their assay could not

280 distinguish the cellular uptake of EVs from functional cargo delivery. To our knowledge,

281 this is the first study demonstrating a feasible real-time assay for cytoplasmic cargo

282 delivery by EVs.

283 Compared to the previously reported assays, the EVCD assay is currently the

284 only method to directly measure the cargo delivery by EVs in living cells. Moreover,

285 the EVCD assay reflected the membrane fusion activity of VSV-G (Fig. 2) and the

286 effect of entry inhibitors (Fig. 3). These results proved the accuracy and feasibility of

287 the assay for quantitative assessment of EV cargo delivery. However, other EV cargo

288 delivery assays may have advantages over the EVCD assay in terms of sensitivity and

289 resolution. For example, RNA (guide RNA or gRNA) delivery by EVs can be measured

290 by a reporter assay utilizing CRISPR/Cas9, the so-called CROSS-FIRE system ${ }^{10}$. The

291 CROSS-FIRE system can measure the delivery of functional cargo gRNA by EVs at the 
292 single-cell level using flow cytometry. This assay is highly sensitive to functional cargo

293 delivery since only a single gRNA delivered to the cytoplasm can lead to the functional

294 readout from recipient cells. However, the CROSS-FIRE system requires multiple

295 additions of EVs to recipient cells and several days are required to obtain the functional

296 readout. Another example of the cargo delivery assay is the use of a BlaM protein as a

297 cargo ${ }^{17,24}$. Cre recombinase-mediated reporter assay has also been reported in several

298 studies ${ }^{11,12,31}$. Each assay has its own pros and cons; therefore, comprehensive analysis

299 of EV cargo delivery may expedite the understanding of the mechanism and

300 physiological relevance of EV-mediated cargo delivery.

301 We estimated that approximately $0.1 \mathrm{fmol}$ of HiBiT per $10^{5}$ cells, equivalent to

302 approximately 600 molecules of HiBiT per cell, can be detected by the EVCD assay

303 (Fig 2A). EPN-01 proteins spontaneously form a 60 -subunit nanocage ${ }^{17}$; hence, a

304 single nanocage has 60-HiBiT molecules. As described previously, one EV contains 14

305 EPN-01 nanocages on average ${ }^{17}$. This indicates that a single EV potentially contains

$306840 \mathrm{HiBiT}$ molecules $(14 \times 60=840)$ on average. Together with the estimated

307 sensitivity of the EVCD assay (600-HiBiT/cell), we assumed that only a single event of

308 EV cargo delivery per cell was enough to exceed the detection threshold in the EVCD

309 assay. In spite of the high sensitivity of the EVCD assay, we could not observe cargo 
310 delivery of EVs without co-expressing VSV-G (Fig. 2B). This result suggests that the

311 authentic EVs that do not possess known fusion proteins is not capable of delivering the

312 cargo, at least for the combination of HEK293T-derived EVs in recipient HEK293T. It

313 is debatable whether EV-mediated cargo delivery is more efficient in other

314 combinations of EVs and recipient cells.

315 Fluorescence imaging is usually used to investigate intracellular trafficking of

316 EVs. However, conventional fluorescence imaging of intracellular EVs labeled with

317 fluorescence dyes or fluorescence proteins cannot be used to evaluate cytoplasmic cargo

318 delivery. To overcome the current limitation of fluorescence imaging of EVs, Joshi et al.

319 succeeded in tracing cargo release using fluorescence imaging of the recruitment of

320 fluorescence-labeled galectin or cargo-specific nanobody ${ }^{7}$. Although their

321 comprehensive analysis is informative to decipher the cargo release process of EVs in

322 recipient cells, it is difficult to distinguish the bona fide cargo release from artifacts of

323 galectin recruitment on endosome/lysosomes. Moreover, fluorescence imaging is not

324 feasible for a high-throughput and real-time analysis. Luminescence imaging is more

325 compatible with live cell imaging by avoiding phototoxicity and photobleaching, which

326 are typical issues in live cell imaging. In this study, we succeeded in live cell imaging of

327 an EV cargo delivery (Fig. 5). As discussed above, EPN-01 could form a 60-subunit 
328 nanocage, and clustering of HiBiT in nanocage resulted in superior brightness in the

329 imaging, as demonstrated by GFP clustering in a similar protein nanocage ${ }^{36}$.

330 Taken together, we developed a quantitative cargo delivery assay of EVs,

331 named the EVCD assay. This assay enabled us to assess the cargo delivery of EVs in

332 recipient cells in real-time. Since EVs are thought to be involved in many biological

333 processes, such as intercellular communication between cells, a feasible EVCD assay

334 may provide insight into the physiological relevance of EVs.

335

336 


\section{Methods}

338 Materials

339 Drugs and antibodies used in this study are summarized in Supplementary Table 1.

340 NanoLuc substrates were purchased from Promega. The HiBiT peptide (amino acid

341 sequence: VSGWRLFKKIS) and the DrkBiT peptide (amino acid sequence:

342 VSGWALFKKIS) ${ }^{22}$ were synthesized by GL Biochem.

343 Additionally, the plasmids used are listed in Supplementary Table 2 and will

344 soon be deposited to Addgene. Plasmids were constructed using PCR-based methods

345 (Gibson Assembly ${ }^{37}$ ) and were confirmed by Sanger sequencing.

346

347 Cell culture and transfection

348 Human embryonic kidney-derived HEK293T (RIKEN Cell Bank) cells were maintained

349 in DMEM supplemented with $10 \%(\mathrm{v} / \mathrm{v})$ fetal bovine serum (FBS) and $10 \mu \mathrm{g} / \mathrm{mL}$

350 penicillin-streptomycin. Cells were cultured at $37^{\circ} \mathrm{C}$ under $5 \% \mathrm{CO}_{2}$ in a humidified

351 incubator.

352 One day before the transfection, approximately $2.0 \times 10^{5}$ cells $/ \mathrm{mL}$ of

353 HEK293T cells were seeded in cell culture dishes or multi-well plates. The following

354 day, HEK293T cells were transfected with plasmid DNA using transfection reagent 
355 polyethyleneimine, Transporter 5 Transfection Reagent (Polyscience, Inc.), or branched

356 25-kDa polyethyleneimine (PEI, Sigma). The ratio of transfection reagent to plasmid

357 DNA was 4:1 (weight). After incubation for 20 to $72 \mathrm{~h}$, cells were subjected to the

358 following experiments.

\section{Characterization of HiBiT-fused proteins}

361 The expression of HiBiT-fused proteins was analyzed using the HiBiT quantification

362 assay and western blotting. For the quantification of HiBiT-tagged proteins, HEK293T

363 cells transfected with the HiBiT protein expression plasmid were lysed, and the amount

364 of the HiBiT protein was measured using the Nano Glo HiBiT Lytic Detection System

365 (Promega). As a quantification standard for HiBiT proteins, HiBiT peptides was used.

366 For western blotting, HEK293T cells expressing HiBiT-fused proteins were lysed with

367 RIPA buffer containing protease inhibitor cocktail (Nacalai Tesque) and separated by

368 SDS-PAGE. After the blotting of proteins on nitrocellulose membranes, HiBiT-fused

369 proteins were visualized using the Nano-Glo HiBiT Blotting System (Promega). As an

370 internal control of HiBiT proteins, GAPDH in the cell lysate was detected using a

371 conventional western blotting protocol using the same membrane as that used for HiBiT

372 detection. 
374 PEG precipitation of EVs containing the HiBiT-tagged protein cargo

375 After 48 to $96 \mathrm{~h}$ of transfection, the supernatant from HEK293T expressing cargo

376 HiBiT proteins was collected, centrifuged at 1,500 $\times g$ for $5 \mathrm{~min}$, mixed with one-third

377 volume of $4 \times$ polyethylene glycol solution (40 w/v\%-PEG6000, $1.2 \mathrm{M}-\mathrm{NaCl}, 1 \times \mathrm{PBS}$

378 [pH 7.4]), and incubated at $4^{\circ} \mathrm{C}$ overnight. The following day, the supernatant was

379 centrifuged at $1,600 \times g$ for $60 \mathrm{~min}$, and the residual pellet was resuspended in PBS.

380 Typically, approximately 5 to $10 \mathrm{~mL}$ of the supernatant was concentrated to 100 to 200

$381 \mu \mathrm{L}$ of PBS (approximately 50-fold concentration). The amount of HiBiT proteins in the

382 concentrated EV fraction was measured using the Nano Glo HiBiT Lytic Detection

383 System (Promega). EV marker proteins in the concentrated EV fraction were detected

384 by western blotting as described above.

386 Live cell extracellular vesicle cargo delivery (EVCD) assay

387 Before 24 to $48 \mathrm{~h}$ of performing the assay, HEK293T cells (1.0 to $2.0 \times 10^{4}$ cells/well)

388 seeded in a PEI-coated 96-well white plate were transfected with the LgBiT-expressing

389 plasmid. For the EVCD assay, the culture medium of LgBiT-expressing HEK293T cells

390 was replaced with HBSS (+) buffer containing $1 \mu \mathrm{M}$ DrkBiT, a peptide that 
391 complements LgBiT and inactivates LgBiT to reduce the background luminescence

392 signal $^{22}$. After the addition of the NanoLuc substrate Nano-Glo Live Cell Assay System

393 (Promega) to the cells, a PEG-concentrated EV fraction (approximately 20 to 100

394 fmol-total HiBiT/well) was added to the cells and monitored for up to $90 \mathrm{~min}$. For the

395 evaluation of inhibitors in the EVCD assay, recipient cells were pretreated with the

396 compounds $1 \mathrm{~h}$ before the assay and treatment with the drugs was continued throughout

397 the assay. Microplate of recipient cells was incubated at $37^{\circ} \mathrm{C}$ and luminescence signal

398 from cells was continually measured by using the Synergy 2 (BioTek) plate reader.

399

400 Live cell luminescence imaging of HiBiT cargo delivery by EVs

401 For luminescence imaging, HEK293T cells (approximately $1.0 \times 10^{4}$ cells/well) were

402 seeded in a poly-L-lysine (PLL)-coated 35-mm multi-well dish (Matsunami Glass Ind.,

403 Ltd.). The following day, cells were transfected with the LgBiT-expressing plasmid and

404 cultured for 24 to 48 h. For live cell imaging, transfected HEK293T cells were washed

405 with $\operatorname{HBSS}(+)$ twice and stored in $\operatorname{HBSS}(+)$ buffer containing $1 \mu \mathrm{M}$ DrkBiT and the

406 NanoLuc substrate, followed by the addition of PEG-concentrated EVs (approximately

40735 fmol-total HiBiT/well). Continuous live cell imaging was carried out using the

408 software MetaMorph and luminescence microscope LV200 (Olympus) equipped with a 
$409100 \times$ objective (Olympus, UPlanSApo, NA $=1.4$ ), a $0.5 \times$ relay lens, and an EM-CCD

410 camera, at $37^{\circ} \mathrm{C}$. The exposure time for each capture was set at $60 \mathrm{~s}$.

411

412 Statistical analysis

413 The data in this work were analyzed using one-way ANOVA and post hoc Dunnett's

414 test or the Student's $t$-test. All statistical analyses were performed using the Real

415 Statistics Resource Pack software developed by Charles Zaiontz.

416

417 Supplementary Files

418 Supplementary Data: Supplementary Methods, Fig. S1, and S2

$419 \quad$ Supplementary Methods

420 Fig. S1: Immunoprecipitation of EVs containing HiBiT-tagged proteins

$421 \quad$ Fig. S2: Requirement of DrkBiT peptide in EVCD assay

422 - Supplementary Tables: Table S1 and S2

423 Table S1: Materials used in this study

424 Table S2: Plasmids used in this study 
425 - Supplementary Video

426 Time-lapse luminescence imaging of recipient HEK293T cells treated with EVs

427 incorporating VSV-G and HiBiT-tagged EPN-01

428

429 Acknowledgements

430 We extend our gratitude to Yumi Yukawa for technical assistance in plasmid

431 construction. Experiments using LV200 were supported by Profs. Takeharu Nagai and

432 Mitsuru Hattori at ISIR, Osaka University. All illustrations in this work were created

433 using BioRender.com.

434 This work was supported in part by JSPS KAKENHI (Grant-in-Aid for

435 Early-Career Scientists 18K18386 and 20K15790 to MS), Research Grant from

436 JGC-Scholarship (to MS), and the "Dynamic Alliance for Open Innovation Bridging

437 Human, Environment and Materials" (MEXT). 


\section{References}

441 (1) Kalluri, R.; LeBleu, V. S. The Biology, Function, and Biomedical Applications

442 of Exosomes. Science 2020, 367 (6478), eaau6977.

443 https://doi.org/10.1126/science.aau6977.

444 (2) Valadi, H.; Ekström, K.; Bossios, A.; Sjöstrand, M.; Lee, J. J.; Lötvall, J. O.

445 Exosome-Mediated Transfer of MRNAs and MicroRNAs Is a Novel Mechanism of

446 Genetic Exchange between Cells. Nat. Cell Biol. 2007, 9 (6), 654-659.

447 https://doi.org/10.1038/ncb1596.

448 (3) Alvarez-Erviti, L.; Seow, Y.; Yin, H.; Betts, C.; Lakhal, S.; Wood, M. J. A.

449 Delivery of SiRNA to the Mouse Brain by Systemic Injection of Targeted Exosomes.

450 Nat. Biotechnol. 2011, 29 (4), 341-345. https://doi.org/10.1038/nbt.1807.

451 (4) Bliss, C. M.; Parsons, A. J.; Nachbagauer, R.; Hamilton, J. R.; Cappuccini, F.;

452 Ulaszewska, M.; Webber, J. P.; Clayton, A.; Hill, A. V. S.; Coughlan, L. Targeting

453 Antigen to the Surface of EVs Improves the In Vivo Immunogenicity of Human and

454 Non-Human Adenoviral Vaccines in Mice. Mol. Ther. - Methods Clin. Dev. 2020, 16,

455 108-125. https://doi.org/10.1016/j.omtm.2019.12.003.

456 (5) Russell, A. E.; Sneider, A.; Witwer, K. W.; Bergese, P.; Bhattacharyya, S. N.;

457 Cocks, A.; Cocucci, E.; Erdbrügger, U.; Falcon-Perez, J. M.; Freeman, D. W.; 
458 Gallagher, T. M.; Hu, S.; Huang, Y.; Jay, S. M.; Kano, S.; Lavieu, G.; Leszczynska, A.;

459 Llorente, A. M.; Lu, Q.; Mahairaki, V.; Muth, D. C.; Noren Hooten, N.; Ostrowski, M.;

460 Prada, I.; Sahoo, S.; Schøyen, T. H.; Sheng, L.; Tesch, D.; Van Niel, G.;

461 Vandenbroucke, R. E.; Verweij, F. J.; Villar, A. V.; Wauben, M.; Wehman, A. M.; Yin,

462 H.; Carter, D. R. F.; Vader, P. Biological Membranes in EV Biogenesis, Stability,

463 Uptake, and Cargo Transfer: An ISEV Position Paper Arising from the ISEV

464 Membranes and EVs Workshop. J. Extracell. Vesicles 2019, 8 (1), 1684862.

465 https://doi.org/10.1080/20013078.2019.1684862.

466 (6) Mulcahy, L. A.; Pink, R. C.; Carter, D. R. F. Routes and Mechanisms of

467 Extracellular Vesicle Uptake. J. Extracell. Vesicles 2014, 3 (1), 1-14.

468 https://doi.org/10.3402/jev.v3.24641.

469 (7) Joshi, B. S.; de Beer, M. A.; Giepmans, B. N. G.; Zuhorn, I. S. Endocytosis of

470 Extracellular Vesicles and Release of Their Cargo from Endosomes. ACS Nano 2020,

471 acsnano.9b10033. https://doi.org/10.1021/acsnano.9b10033.

472 (8) Montecalvo, A.; Larregina, A. T.; Shufesky, W. J.; Stolz, D. B.; Sullivan, M. L.

473 G.; Karlsson, J. M.; Baty, C. J.; Gibson, G. A.; Erdos, G.; Wang, Z.; Milosevic, J.;

474 Tkacheva, O. A.; Divito, S. J.; Jordan, R.; Lyons-Weiler, J.; Watkins, S. C.; Morelli, A.

475 E. Mechanism of Transfer of Functional MicroRNAs between Mouse Dendritic Cells 
476 via Exosomes. Blood 2012, 119 (3), 756-766.

477 https://doi.org/10.1182/blood-2011-02-338004.

478 (9) Somiya, M. Where Does the Cargo Go?: Solutions to Provide Experimental

479 Support for the "Extracellular Vesicle Cargo Transfer Hypothesis.” J. Cell Commun.

480 Signal. 2020. https://doi.org/10.1007/s12079-020-00552-9.

481 (10) de Jong, O. G.; Murphy, D. E.; Mäger, I.; Willms, E.; Garcia-Guerra, A.;

482 Gitz-Francois, J. J.; Lefferts, J.; Gupta, D.; Steenbeek, S. C.; van Rheenen, J.; El

483 Andaloussi, S.; Schiffelers, R. M.; Wood, M. J. A.; Vader, P. A CRISPR-Cas9-Based

484 Reporter System for Single-Cell Detection of Extracellular Vesicle-Mediated

485 Functional Transfer of RNA. Nat. Commun. 2020, 11 (1).

486 https://doi.org/10.1038/s41467-020-14977-8.

487 (11) Zomer, A.; Maynard, C.; Verweij, F. J.; Kamermans, A.; Schäfer, R.; Beerling,

488 E.; Schiffelers, R. M.; de Wit, E.; Berenguer, J.; Ellenbroek, S. I. J.; Wurdinger, T.;

489 Pegtel, D. M.; van Rheenen, J. In Vivo Imaging Reveals Extracellular Vesicle-Mediated

490 Phenocopying of Metastatic Behavior. Cell 2015, 161 (5), 1046-1057.

491 https://doi.org/10.1016/j.cell.2015.04.042. 
492 (12) Sterzenbach, U.; Putz, Uu.; Low, L.-H.; Silke, J.; Tan, S.-S.; Howitt, J.

493 Engineered Exosomes as Vehicles for Biologically Active Proteins. Mol. Ther. 2017, 25

494 (6), 1-10. https://doi.org/10.1016/j.ymthe.2017.03.030.

495 (13) Whittaker, T. E.; Nagelkerke, A.; Nele, V.; Kauscher, U.; Stevens, M. M.

496 Experimental Artefacts Can Lead to Misattribution of Bioactivity from Soluble

497 Mesenchymal Stem Cell Paracrine Factors to Extracellular Vesicles. J. Extracell.

498 Vesicles 2020, 9 (1), 1807674. https://doi.org/10.1080/20013078.2020.1807674.

499 (14) Jin, Y.; Chen, Z.; Liu, X.; Zhou, X. Evaluating the MicroRNA Targeting Sites

500 by Luciferase Reporter Gene Assay. In MicroRNA Protocols; Ying, S.-Y., Ed.; Methods

501 in Molecular Biology; Humana Press: Totowa, NJ, 2013; Vol. 936, pp 117-127.

502 https://doi.org/10.1007/978-1-62703-083-0_10.

503 (15) Dixon, A. S.; Schwinn, M. K.; Hall, M. P.; Zimmerman, K.; Otto, P.; Lubben,

504 T. H.; Butler, B. L.; Binkowski, B. F.; Machleidt, T.; Kirkland, T. A.; Wood, M. G.;

505 Eggers, C. T.; Encell, L. P.; Wood, K. V. NanoLuc Complementation Reporter

506 Optimized for Accurate Measurement of Protein Interactions in Cells. ACS Chem. Biol.

507 2016, 11 (2), 400-408. https://doi.org/10.1021/acschembio.5b00753.

508 (16) Hall, M. P.; Unch, J.; Binkowski, B. F.; Valley, M. P.; Butler, B. L.; Wood, M.

509 G.; Otto, P.; Zimmerman, K.; Vidugiris, G.; MacHleidt, T.; Robers, M. B.; Benink, H. 
510 A.; Eggers, C. T.; Slater, M. R.; Meisenheimer, P. L.; Klaubert, D. H.; Fan, F.; Encell, L.

511 P.; Wood, K. V. Engineered Luciferase Reporter from a Deep Sea Shrimp Utilizing a

512 Novel Imidazopyrazinone Substrate. ACS Chem. Biol. 2012.

513 https://doi.org/10.1021/cb3002478.

514 (17) Votteler, J.; Ogohara, C.; Yi, S.; Hsia, Y.; Nattermann, U.; Belnap, D. M.;

515 King, N. P.; Sundquist, W. I. Designed Proteins Induce the Formation of

516 Nanocage-Containing Extracellular Vesicles. Nature 2016, 540 (7632), 292-295.

517 https://doi.org/10.1038/nature20607.

518 (18) Rider, M. A.; Hurwitz, S. N.; Meckes, D. G. ExtraPEG: A Polyethylene

519 Glycol-Based Method for Enrichment of Extracellular Vesicles. Sci. Rep. 2016, 6 (1).

520 https://doi.org/10.1038/srep23978.

521 (19) Meyer, C.; Losacco, J.; Stickney, Z.; Li, L.; Marriott, G.; Lu, B. Pseudotyping

522 Exosomes for Enhanced Protein Delivery in Mammalian Cells. Int. J. Nanomedicine

$5232017,12,3153-3170$. https://doi.org/10.2147/IJN.S133430.

524 (20) Johannsdottir, H. K.; Mancini, R.; Kartenbeck, J.; Amato, L.; Helenius, A.

525 Host Cell Factors and Functions Involved in Vesicular Stomatitis Virus Entry. J. Virol.

5262009,83 (1), 440-453. https://doi.org/10.1128/JVI.01864-08. 
527 (21) Le Blanc, I.; Luyet, P.-P.; Pons, V.; Ferguson, C.; Emans, N.; Petiot, A.;

528 Mayran, N.; Demaurex, N.; Fauré, J.; Sadoul, R.; Parton, R. G.; Gruenberg, J.

529 Endosome-to-Cytosol Transport of Viral Nucleocapsids. Nat. Cell Biol. 2005, 7 (7),

530 653-664. https://doi.org/10.1038/ncb1269.

531 (22) Yamamoto, M.; Du, Q.; Song, J.; Wang, H.; Watanabe, A.; Tanaka, Y.;

532 Kawaguchi, Y.; Inoue, J.; Matsuda, Z. Cell-Cell and Virus-Cell Fusion Assay-Based

533 Analyses of Alanine Insertion Mutants in the Distal A9 Portion of the JRFL Gp41

534 Subunit from HIV-1. J. Biol. Chem. 2019, 294 (14), 5677-5687.

535 https://doi.org/10.1074/jbc.RA118.004579.

536 (23) Fredericksen, B. L.; Whitt, M. A. Vesicular Stomatitis Virus Glycoprotein

537 Mutations That Affect Membrane Fusion Activity and Abolish Virus Infectivity. $J$.

538 Virol. 1995, 69 (3), 1435-1443. https://doi.org/10.1128/JVI.69.3.1435-1443.1995.

539 (24) Albanese, M.; Chen, Y.-F. A.; Huels, C.; Gaertner, K.; Tagawa, T.; Keppler, O.

540 T.; Goebel, C.; Zeidler, R.; Hammerschmidt, W. Micro RNAs Are Minor Constituents of

541 Extracellular Vesicles and Are Hardly Delivered to Target Cells; bioRxiv; preprint;

542 Cell Biology, 2020. https://doi.org/10.1101/2020.05.20.106393.

543 (25) Le Saux, S.; Aarrass, H.; Lai-Kee-Him, J.; Bron, P.; Armengaud, J.; Miotello,

544 G.; Bertrand-Michel, J.; Dubois, E.; George, S.; Faklaris, O.; Devoisselle, J.-M.; 
545 Legrand, P.; Chopineau, J.; Morille, M. Post-Production Modifications of Murine

546 Mesenchymal Stem Cell (MMSC) Derived Extracellular Vesicles (EVs) and Impact on

547 Their Cellular Interaction. Biomaterials 2020, 231, 119675.

548 https://doi.org/10.1016/j.biomaterials.2019.119675.

549 (26) Pastuzyn, E. D.; Day, C. E.; Kearns, R. B.; Kyrke-Smith, M.; Taibi, A. V.;

550 McCormick, J.; Yoder, N.; Belnap, D. M.; Erlendsson, S.; Morado, D. R.; Briggs, J. A.

551 G.; Feschotte, C.; Shepherd, J. D. The Neuronal Gene Arc Encodes a Repurposed

552 Retrotransposon Gag Protein That Mediates Intercellular RNA Transfer. Cell 2018, 172

553 (1-2), 275-288.e18. https://doi.org/10.1016/j.cell.2017.12.024.

554 (27) Yao, Z.; Qiao, Y.; Li, X.; Chen, J.; Ding, J.; Bai, L.; Shen, F.; Shi, B.; Liu, J.;

555 Peng, L.; Li, J.; Yuan, Z. Exosomes Exploit the Virus Entry Machinery and Pathway To

556 Transmit Alpha Interferon-Induced Antiviral Activity. J. Virol. 2018, 92 (24),

557 e01578-18, /jvi/92/24/e01578-18.atom. https://doi.org/10.1128/JVI.01578-18.

558 (28) Horibe, S.; Tanahashi, T.; Kawauchi, S.; Murakami, Y.; Rikitake, Y.

559 Mechanism of Recipient Cell-Dependent Differences in Exosome Uptake. BMC Cancer

560 2018, 18 (1), 47. https://doi.org/10.1186/s12885-017-3958-1.

561 (29) Colpitts, T. M.; Moore, A. C.; Kolokoltsov, A. A.; Davey, R. A. Venezuelan

562 Equine Encephalitis Virus Infection of Mosquito Cells Requires Acidification as Well 
563 as Mosquito Homologs of the Endocytic Proteins Rab5 and Rab7. Virology 2007, 369

564 (1), 78-91. https://doi.org/10.1016/j.virol.2007.07.012.

565 (30) Yonezawa, A.; Cavrois, M.; Greene, W. C. Studies of Ebola Virus

566 Glycoprotein-Mediated Entry and Fusion by Using Pseudotyped Human

567 Immunodeficiency Virus Type 1 Virions: Involvement of Cytoskeletal Proteins and

568 Enhancement by Tumor Necrosis Factor Alpha. J. Virol. 2005, 79 (2), 918-926.

569 https://doi.org/10.1128/JVI.79.2.918-926.2005.

570 (31) Heath, N.; Osteikoetxea, X.; de Oliveria, T. M.; Lázaro-Ibáñez, E.; Shatnyeva,

571 O.; Schindler, C.; Tigue, N.; Mayr, L. M.; Dekker, N.; Overman, R.; Davies, R.

572 Endosomal Escape Enhancing Compounds Facilitate Functional Delivery of

573 Extracellular Vesicle Cargo. Nanomed. 2019, 14 (21), 2799-2814.

574 https://doi.org/10.2217/nnm-2019-0061.

575 (32) Sasaki, M.; Anindita, P. D.; Phongphaew, W.; Carr, M.; Kobayashi, S.; Orba,

576 Y.; Sawa, H. Development of a Rapid and Quantitative Method for the Analysis of

577 Viral Entry and Release Using a NanoLuc Luciferase Complementation Assay. Virus

578 Res. 2018, 243, 69-74. https://doi.org/10.1016/j.virusres.2017.10.015.

579 (33) Miyakawa, K.; Jeremiah, S. S.; Ohtake, N.; Matsunaga, S.; Yamaoka, Y.; Nishi,

580 M.; Morita, T.; Saji, R.; Nishii, M.; Kimura, H.; Hasegawa, H.; Takeuchi, I.; Ryo, A. 


\section{Rapid Quantitative Screening Assay for SARS-CoV-2 Neutralizing Antibodies Using}

582 HiBiT-Tagged Virus-like Particles; preprint; Infectious Diseases (except HIV/AIDS),

583 2020. https://doi.org/10.1101/2020.07.20.20158410.

584 (34) Evans, B. C.; Fletcher, R. B.; Kilchrist, K. V.; Dailing, E. A.; Mukalel, A. J.;

585 Colazo, J. M.; Oliver, M.; Cheung-Flynn, J.; Brophy, C. M.; Tierney, J. W.; Isenberg, J.

586 S.; Hankenson, K. D.; Ghimire, K.; Lander, C.; Gersbach, C. A.; Duvall, C. L. An

587 Anionic, Endosome-Escaping Polymer to Potentiate Intracellular Delivery of Cationic

588 Peptides, Biomacromolecules, and Nanoparticles. Nat. Commun. 2019, 10 (1), 5012.

589 https://doi.org/10.1038/s41467-019-12906-y.

590 (35) Toribio, V.; Morales, S.; López-Martín, S.; Cardeñes, B.; Cabañas, C.;

591 Yáñez-Mó, M. Development of a Quantitative Method to Measure EV Uptake. Sci. Rep.

592 2019, 9 (1), 10522. https://doi.org/10.1038/s41598-019-47023-9.

593 (36) Hsia, Y.; Bale, J. B.; Gonen, S.; Shi, D.; Sheffler, W.; Fong, K. K.; Nattermann,

594 U.; Xu, C.; Huang, P.-S.; Ravichandran, R.; Yi, S.; Davis, T. N.; Gonen, T.; King, N.

595 P.; Baker, D. Design of a Hyperstable 60-Subunit Protein Icosahedron. Nature 2016,

596535 (7610), 136-139. https://doi.org/10.1038/nature18010. 
597 (37) Gibson, D. G.; Young, L.; Chuang, R.-Y.; Venter, J. C.; Hutchison, C. A.;

598 Smith, H. O. Enzymatic Assembly of DNA Molecules up to Several Hundred Kilobases.

599 Nat. Methods 2009, 6 (5), 343-345. https://doi.org/10.1038/nmeth.1318.

600 